\title{
Die Würde des Menschen - AEM Jahrestagung 2016
}

\author{
Ralf Stoecker
}

Online publiziert: 10. Mai 2016

(C) Springer-Verlag Berlin Heidelberg 2016

Die Würde des Menschen ist unantastbar. Sie zu achten und zu schützen ist Verpflichtung aller staatlichen Gewalt. Das Deutsche Volk bekennt sich darum zu unverletzlichen und unveräußerlichen Menschenrechten als Grundlage jeder menschlichen Gemeinschaft, des Friedens und der Gerechtigkeit in der Welt.

(Grundgesetz, Art. 1)

Es hat eine Weile gedauert, bis die Philosophie die Herausforderung erkannt und angenommen hat, die in Artikel 1 des Grundgesetzes (GG) steckt. Sie besteht darin, dass in diesen Sätzen kurz und bündig eine Antwort auf die traditionelle Masterfrage der Moralphilosophie gegeben wird, worin die Grundlagen unserer wechselseitigen ethischen Verpflichtungen liegen: Es ist die unantastbare Menschenwürde, die die Basis für Menschenrechte, Sozialleben, Frieden und Gerechtigkeit bildet. Dadurch wird ein Begriff, der zwar eine philosophische Tradition und mit Kant einen hochkarätigen Befürworter hat, aber auch durch Schopenhauer heftig kritisiert und danach allenfalls am Rande philosophisch diskutiert wurde, mit einem juristischen Federstrich zum Zentrum unseres moralischen Status erklärt.

Wie gesagt, die Philosophie hat diese juristische Zumutung lange Zeit weitgehend ignoriert, anders als die Rechtswissenschaft, die natürlich nicht umhin konnte, sich mit dem Fundament unserer Verfassung auseinanderzusetzen. Philosophisch auffällig wurde die besondere Rolle der Menschenwürde im Grundgesetz eigentlich erst gegen Ende des Jahrtausends, und zwar in einem Zusammenhang, der mit dem ursprünglichen Anliegen von Artikel 1 herzlich wenig zu tun hatte: in der medizinethischen Diskussion um die Zulässigkeit von Stammzellforschung und Präimplantationsdiagnostik. Beide Verfahren bringen es mit sich, dass Embryonen getötet

Prof. Dr. R. Stoecker $(\bowtie)$

Abteilung Philosophie, Universität Bielefeld

33501 Bielefeld, Deutschland

E-Mail: ralf.stoecker@uni-bielefeld.de 
werden, also stellte sich die Frage, ob nicht auch schon der Embryo eine unantastbare Menschenwürde hat und damit unter das Gebot aus Artikel 1 fällt, seine Würde zu achten und ihn folglich nicht einfach zu vernichten. Um das zu beantworten, musste man aber wissen, was es ganz generell mit der Menschenwürde auf sich hat. So kam es dann um die Jahrtausendwende zu einer scharf geführten, sehr kontroversen Auseinandersetzung zwischen Befürwortern und Gegnern der Embryonenwürde, die in der Regel zugleich Gegner bzw. Befürworter der entsprechenden medizinischen Technologien waren. Ein klares Bild, was es denn eigentlich mit der Würde des Menschen auf sich hat, ergab sich aus diesen Debatten aber nicht.

Seitdem hat sich die philosophische Diskussion erheblich weiterentwickelt. In den letzten Jahren sind eine Vielzahl von Publikationen erschienen, die davon zeugen. ${ }^{1}$ Was diese Publikationen zeigen ist zweierlei: Erstens, die Menschenwürde ist interessanter als es den Philosophen lange Zeit erschienen ist, und zweitens, sie ist auch rätselhafter. Das heißt, es lohnt sich zwar, sich ethisch mit der Menschenwürde zu beschäftigen, es ist aber auch nicht ganz leicht. Das ist der Hintergrund, vor dem im Herbst in Bielefeld die Jahrestagung der AEM zu dem Thema „Auf Augenhöhe Zur Bedeutung der Menschenwürde für Medizin und Gesundheitswesen“"stattfindet, für die ich in diesem Editorial werben möchte.

Der umgangssprachliche Obertitel „Auf Augenhöhe“ zeigt schon, dass das Thema keineswegs nur für die philosophische Grundlagenforschung interessant ist. Ganz im Gegenteil! Es ist eine der Eigentümlichkeiten der Menschenwürde, dass das Konzept der Menschenwürde zwar philosophisch (und auch juristisch) immer wieder Skepsis und Misstrauen hervorgerufen hat, in der Praxis ethischer Bewertungen aber schon lange weit verbreitet war und bis heute ist. Spätestens seit dem 19. Jahrhundert werden politische und soziale Missstände, aber auch einzelne persönliche Verfehlungen mit dem Rückgriff auf die Menschenwürde angeprangert. Dabei muss auch nicht immer ausdrücklich von „Würde“ die Rede sein. Es gibt einen ganzen Strauß von Ausdrücken und Redewendungen, mit denen wir uns auf die Würde und ihre Verletzungen beziehen. Wir reden von Menschlichkeit und Unmenschlichkeit, Demütigungen und Erniedrigungen, Selbstachtung und Gesichtsverlust, Menschen werden respektiert oder klein gemacht, man begegnet ihnen mit Verachtung oder eben auf Augenhöhe usw., und anscheinend versteht man ganz gut, was damit gemeint ist. In diesem Sinn ist uns die menschliche Würde häufig gar nicht rätselhaft; nur beim näheren Hinsehen, in der ethischen Reflexion, verschwimmt das Bild plötzlich.

Diese Spannung zwischen der vermeintlichen Vertrautheit und inhaltlichen Ungreifbarkeit bildet nur eine der Widersprüchlichkeiten, denen man begegnet, wenn man sich näher mit der Menschenwürde beschäftigt. Eine andere ist bereits in der Formulierung von Art. 1 GG angelegt: zwischen einerseits der Unantastbarkeit und andererseits der offenkundigen Verletzlichkeit der Menschenwürde. Zweifellos kann die Würde angetastet werden, ebenso zweifellos geht das Grundgesetz aber davon aus, dass diese Verletzung die Würde der Opfer nicht mindert. Wie ist das möglich?

\footnotetext{
1 In Deutschland u. a. Joerden et al. [7]; Gröschner et al. [5]; Bieri [3]; Hilgendorf [6], Schaber [9, 10], Bielefeldt [2]; international u. a. Düwell et al. [4]; Rosen [8]; Waldron [12]; The President's Council of Bioethics [11]. Zur Rolle der Würde in der Pflege vgl. auch die Sammelrezension Baranzke [1].
} 
Damit hängt eine andere Spannung zusammen, zwischen der Würde der Opfer und der der Täter. Gerade bei paradigmatischen schweren Menschenwürdeverletzungen wird immer wieder behauptet, dass es nicht eigentlich die Opfer seien, die dadurch ihre Würde aufs Spiel setzten, sondern die Täter, die sich so unwürdig verhielten. Nicht der im KZ zu Tode gequälte Pastor Maximilian Kolbe habe seine Würde verloren, so hat es der Philosoph Robert Spaemann einmal ausgedrückt, sondern der KZ-Aufseher, der ihm dies angetan hat. Dem steht allerdings entgegen, dass es natürlich nicht die Sorge um die Würde der SS-Männer war, die dazu geführt hat, nach dem Zweiten Weltkrieg die Menschenwürde in die Allgemeine Erklärung der Menschenrechte und das Grundgesetz aufzunehmen, sondern der Eindruck der massiven Verletzungen der Würde ihrer Opfer.

Ein weiterer Widerspruch betrifft den elitären Charakter des Würdebegriffs. Würden sind traditionell dasjenige, was manche Menschen vor anderen auszeichnet, sei es, dass sie sie erworben haben oder dass eine hierarchisch aufgebaute Gesellschaft sie ihnen auch ohne Verdienste zuerkennt. Wie aber kann man dann von einer für alle Menschen gleichen Menschenwürde reden? Ist das nicht, wie Mitte des 19. Jahrhunderts Arthur Schopenhauer geargwöhnt hat, ein Widerspruch in sich? Oder soll damit bloß speziesistisch ein Vorrang des Menschen vor dem Tier formuliert werden?

Hinzu kommt der Verdacht, dass es sich bei der Würde ohnehin um ein längst überholtes Ideal handelt, eine verkrampfte Förmlichkeit, die überhaupt nicht mehr in unsere Zeit der Freizügigkeit, individuellen Lässigkeit und ironischen Brechung sozialer Konventionen passt. Schließlich haben uns erst vor ein paar Monaten die Morde in Paris daran erinnert, wie wichtig die Respektlosigkeiten engagierter Journalisten in unserer Gesellschaft sind. Wie kann man dann noch annehmen, dass es unsere Würde sei, in der unser moralischer Wert zu finden ist? - Dem stehen wiederum die aktuellen Bilder aus Flüchtlingscamps entgegen, bei denen man sich unmittelbar fragt, wie es mit der Menschenwürde vereinbar sein könne, diese Menschen in Kälte, Schlamm und Hunger alleine zu lassen, ohne dass dies irgendetwas mit Förmlichkeit zu tun hätte. Und auch in der medizinischen Ethik beklagen wir den menschenunwürdigen Umgang mit alten und schwachen Menschen oder streiten darum, ob es zum Sterben in Würde gehören könnte, selbstbestimmt seinem Leben ein Ende zu setzen, ohne das Gefühl zu haben, antiquierten Vorstellungen von Ehrwürdigkeit anzuhängen.

Die moderne Philosophie bietet natürlich eine ganze Reihe von Erklärungen und Lösungsvorschlägen für die Spannungen und Widersprüche, mit denen uns der Begriff der Menschenwürde auf den ersten Blick konfrontiert. Sie unterscheidet beispielsweise verschiedene Formen der Würde, bietet Reduktionen des Würdebegriffs auf andere, vertrautere ethische Konzeptionen oder argwöhnt, dass sich der Verweis auf die Menschenwürde letztlich als inhaltsleerer Ausdruck moralischen Unbehagens entpuppen könnte. Die Debatte ist noch weit offen, sie hat gerade erst begonnen. Nach unserer Überzeugung lohnt es sich jedenfalls, sie intensiv zu führen. Die Vision, die nach dem Zweiten Weltkrieg und der Nazizeit die Autoren und (sehr wenigen) Autorinnen des Grundgesetzes dazu geführt hat, an den Anfang der deutschen Verfassung eine Antwort auf die moralphilosophische Masterfrage zu stellen, könnte auch noch siebzig Jahre später zu überraschenden ethischen Einsichten führen zum Beispiel im September in Bielefeld. 


\section{Literatur}

1. Baranzke H (2015) Sammelrezension. Ethik Med 27(4):355-358

2. Bielefeldt H (2011) Auslaufmodell Menschenwürde? Warum sie in Frage steht und warum wir sie verteidigen müssen. Herder, Freiburg i. Br. u. a.

3. Bieri P (2013) Eine Art zu leben. Über die Vielfalt menschlicher Würde. Carl Hanser Verlag, München

4. Düwell M, Braarvig J, Brownsword R, Mieth D (Hrsg) (2014) The Cambridge handbook of human dignity. Interdisciplinary perspectives. Cambridge Univ. Press, Cambridge

5. Gröschner R, Kapust A, Lembcke OW (Hrsg) (2013) Wörterbuch der Würde. Fink, München

6. Hilgendorf E (Hrsg) (2013) Menschenwürde und Demütigung. Die Menschenwürdekonzeption Avishai Margalits. Nomos, Baden-Baden

7. Joerden JC, Hilgendorf E, Thiele F (Hrsg) (2013) Menschenwürde und Medizin. Ein interdisziplinäres Handbuch. Duncker \& Humblot, Berlin

8. Rosen M (2012) Dignity. Its history and meaning. Harvard University Press, Cambridge Mass

9. Schaber P (2010) Instrumentalisierung und Würde. mentis, Paderborn

10. Schaber P (2012) Menschenwürde. Grundwissen Philosophie. Philipp Reclam, Ditzingen

11. The President's Council of Bioethics (Hrsg) (2008) Human dignity and bioethics. Washington DC

12. Waldron J (2012) Dignity, rank, and rights. Oxford Univ. Press, Oxford u. a. 